\title{
Positive and negative transfer of conditioned aversive stimuli to a conditioned appetitive excitor as a function of aversive US intensity
}

\author{
PAUL L. DEVITO \\ Saint Joseph's University, Philadelphia, Pennsylvania \\ and \\ HARRY FOWLER \\ University of Pittsburgh, Pittsburgh, Pennsylvania
}

\begin{abstract}
Rats were given Pavlovian aversive (Av) conditioning in which a weak (0.5-sec, $0.7-\mathrm{mA}$ ) or a strong (0.5-sec, 1.4-mA) footshock unconditioned stimulus (US) was presented alone or in a positive, zero, or negative correlation with a flashing-light conditioned stimulus (AvCS+, AvCSo, or AvCS-, respectively). Thereafter, the subjects received Pavlovian appetitive (Ap) conditioning in which the flashing-light CS was positively correlated in a forward order with the delivery of a food US. As anticipated, for subjects that had been trained with the strong shock US, the AvCS+ retarded and the AvCS- facilitated Ap conditioning relative to both the AvCS $o$ and the novel-CS treatments, which did not differ in effect. However, the exact opposite prevailed for the AvCS+ and AvCS- subjects that had been trained with the weak shock US. On the basis of these and other data, we propose an "ABC" model of transfer that stresses the importance of affective, behavioral, and cognitive factors in accounting for the divergent results that are obtained in both Av-to-Ap and Ap-to-Av transfer paradigms.
\end{abstract}

Across-reinforcement transfer is a Pavlovian conditioning paradigm in which a conditioned stimulus (CS) is first associated with either an aversive (Av) unconditioned stimulus (US) or an appetitive (Ap) US and then is subsequently conditioned with the opposite US (see, e.g., Dickinson \& Pearce, 1977). Previous research on Av-toAp transfer has uniformly shown "negative transfer" in which prior excitatory conditioning of a CS as a signal, say, for shock (AvCS+) retards the subsequent reconditioning of that CS as a signal, say, for food (ApCS+) (e.g., Bromage \& Scavio, 1978; Kaye, Preston, Szabo, Druiff, \& Mackintosh, 1987; Konorski \& Szwejkowska, 1956; Krank, 1985; Peck \& Bouton, 1990; Scavio, 1974). Conversely, prior inhibitory conditioning of a CS as a signal for the absence of shock (AvCS-) facilitates the CS's transformation into an ApCS+ (e.g., Bromage \& Scavio, 1978; Konorski \& Szwejkowska, 1956; Scavio, 1974).

Previous research on the opposite paradigm, entailing Ap-to-Av transfer, has shown a mixture of outcomes: negative transfer, in which initial ApCS + training retards

The research was supported in part by a Saint Joseph's University Faculty Research grant to P. L. DeVito and by National Institutes of Health Grant MH24115 to H. Fowler. Correspondence should be addressed to P. L. DeVito, Department of Psychology, Saint Joseph's University, 5600 City Avenue, Philadelphia, PA 19131. the CS's transformation into an AvCS+ (e.g., Dickinson, 1976, Experiment 1; Konorski \& Szwejkowska, 1956); null transfer, in which prior ApCS+ training has no effect on subsequent AvCS+ conditioning (e.g., Jackson, 1974; Peck \& Bouton, 1990); and positive transfer, in which initial ApCS+ training facilitates the CS's transformation into an AvCS+ (e.g., DeVito \& Fowler, 1982; Dickinson, 1976, Experiment 2; Scavio \& Gormezano, 1980). Positive Ap-to-Av transfer has also been reported with paradigms involving inhibitory conditioning. In this case, prior ApCS - training facilitates the CS's transformation into an AvCS- (DeVito \& Fowler, 1982).

We previously proposed a signaling interpretation for the positive transfer found in our Ap-to-Av transfer research involving both excitatory and inhibitory conditioning (DeVito \& Fowler, 1982). According to that interpretation, the subject learns that a CS signals either the presence $(\mathrm{CS}+$ ) or absence (CS-) of a US, while also learning that the signaled US is affectively positive (Ap) or affectively negative (Av). Assuming that the affective property of the CS is relatively transient and dependent upon the type of US that is present, we argued that the CS's signaling property would transfer to an affectively opposite US, with the result that excitatory conditioning to the new US is (1) facilitated by prior excitatory conditioning with the opposite US and (2) retarded by prior inhibitory conditioning with the opposite US. Likewise, inhibitory conditioning with the new US should be (1) facil- 
itated by prior inhibitory conditioning with the opposite US and (2) retarded by prior excitatory conditioning with the opposite US.

To reconcile the above interpretation with the seemingly contradictory negative transfer found in the Av-to-Ap paradigm, we suggested that the affective impact of an AvUS is biologically more potent and, thus, more inertial than the affective impact of an ApUS. Accordingly, we reasoned that the negative affect associated with a CS predicting an AvUS would not be altered rapidly in Avto-Ap transfer, since the animal would be subject to the combined influences of the CS's initial signal and affective values. In other words, there would be a relatively persistent fear reaction associated with a signal for the presence of an AvUS, which, in turn, would interfere with the signal's development as an ApCS+. Likewise, we argued that there should be a relatively persistent relief or fear-inhibitory reaction to a signal for the absence of an AvUS, which, in turn, should facilitate the CS's transformation into an ApCS+.

Although our signaling interpretation is still viable, it is based primarily on the instances of positive transfer found in the Ap-to-Av paradigm (e.g., DeVito \& Fowler, 1982; Scavio \& Gormezano, 1980) and, as such, it does not directly (or sufficiently) address the instances of negative transfer found in that paradigm. To account for the divergent and contrasting results, as well as to assimilate the findings of both Ap-to-Av and Av-to-Ap transfer, we want to propose a reinterpretation of the positive transfer findings. This new interpretation is based on a "discrepancy" account of conditioning (e.g., Fowler, 1978; Fowler, Lysle, \& DeVito, 1991; Rescorla \& Wagner, 1972 ) in which a CS is acknowledged to have both affective and cognitive components by which it predicts either a generally "good" or "bad" outcome-namely, that an ApUS or an AvUS is or is not coming. Applied to Ap-toAv transfer, this interpretation argues that an AvUS will produce a large affective discrepancy for subjects who have received ApCS+ training, because the ApCS+ predicts a good outcome (e.g., food) that is highly discrepant from the bad outcome (e.g., shock) that now prevails. In contrast, there will be a relatively small affective discrepancy for subjects who have received ApCS - training, because the ApCS- predicts a bad outcome (i.e., no food), comparable to the bad outcome of shock that is now obtained. Hence, the large discrepancy generated by the ApCS+ can facilitate aversive excitatory conditioning to itself, whereas the small discrepancy produced by the ApCS- can retard (or 'block") such reconditioning. In short, this interpretation argues that the magnitude of the affective discrepancy between what the CS predicts and what the US provides is the primary factor determining the rate and strength of reconditioning of the CS.

Our new interpretation should not be taken to imply that only affective/cognitive aspects of a CS influence transfer, for such an interpretation by itself cannot account for the instances of negative transfer found in the Ap-to-Av paradigm or for the negative transfer uniformly found in the Av-to-Ap paradigm. However, we would argue that, in conjunction with affective/cognitive factors, other factors relating, for example, to the type of behavior that is initially conditioned to the pretreated stimulus (e.g., Holland, 1979) will also be important. This behavioral factor was not crucial in our prior demonstration of positive Ap-to-Av transfer (DeVito \& Fowler, 1982) because the responses conditioned to the ApCS+ and the ApCS- were grossly comparable in that they involved locomotion to the food cup and locomotion in general (e.g., exploration). Accordingly, with these grossly equivalent locomotive behaviors, the subsequent conditioning of suppression to the ApCS via a conditioned-emotional-response (CER) procedure was affected primarily by affective/cognitive factors. On the other hand, in studies that found null or negative transfer in the Ap-to-Av paradigm, it is conceivable that grossly nonequivalent behaviors were conditioned to the different ApCSs and that those behaviors were responsible for the failure to find positive transfer.

By way of illustration, one can easily conceive of a case in which the behavioral and the affective/cognitive factors are different for groups trained with either a CS+ or a CS-, and thus where both sets of factors influence transfer. Consider, for example, a paradigm in which $\mathrm{CS}+$ and CS - groups are initially trained on a CER procedure and then are subjected, with the same CS, to appetitive excitatory conditioning involving locomotion to a food cup. In this Av-to-Ap transfer paradigm, one would expect the subjects trained with the AvCS+ to have a large affective discrepancy (i.e., predicted bad but good outcome), which would facilitate transfer, and a large behavioral discrepancy (e.g., from freezing to approaching the food cup), which would retard transfer. In contrast, for subjects trained with the AvCS-, one would expect a small affective discrepancy (i.e., predicted and obtained good outcome), which would retard transfer, and a small behavioral discrepancy (i.e., from locomoting generally to approaching the food cup), which would facilitate transfer. Thus, depending on the relative weighting of the two dimensions, this type of paradigm could easily generate both positive and negative transfer.

The present study was conducted to evaluate the above possibility. Different groups of rats were first given CER training in which a weak or a strong shock US was presented alone or in a positive, zero, or negative correlation with a 5-sec flashing-light stimulus, so as to establish that stimulus as a novel CS or as an AvCS+, CSo, or CS-, respectively. The use of both weak and strong shock USs in the CER phase was for the purpose of conditioning behaviors of varying strengths with the same CS-US correlations. Afterward, the rate of appetitive excitatory conditioning to the previously established AvCS was assessed by measuring, from the onset of that CS, the subject's latency in contacting the site of delivery of a food US that was presented immediately upon termination of the CS. 


\section{METHOD}

\section{Subjects}

Thirty-two experimentally naive, male albino rats of the SpragueDawley strain, purchased from the Holtzman Co. at 90 days of age and at a weight of approximately $350 \mathrm{~g}$, served as subjects. Upon arrival, and for the duration of the experiment, the subjects were individually caged in a federally certifiable animal colony room where the temperature was maintained at $22^{\circ} \mathrm{C}, 50 \%$ relative humidity, and the 12:12-h day:night cycle was reversed through artificial illumination.

\section{Apparatus}

Two apparatuses were used. Four modified Coulbourn Instruments rodent chambers (Model E10-10), measuring $25 \times 30 \times 33 \mathrm{~cm}$, served as the aversive-conditioning (CER) apparatuses. The chambers were housed in four sound-attenuating cubicles located in a room adjacent to the programming equipment. A $50-\mathrm{ml}$ drinking tube (BRS/LVE Model DT-001), connected through a contact relay to recording circuitry, allowed continuous monitoring of licking behavior in the chamber. A $6-\mathrm{W} 120-\mathrm{V}$ bulb, centered in the ceiling of the test chambers and connected to two interval timers (Hunter Model 111-B), provided a visual CS: a 5-sec light flashing on and off at the rate of $0.4 \mathrm{sec}$ per cycle. The grid floor of each chamber, composed of $0.24-\mathrm{cm}$ bars spaced $0.87 \mathrm{~cm}$ apart, was connected through timer circuitry to the output of a BRS/LVE shock scrambler (Model SC 902) and generator (Model 903) to provide an aversive US: either a $0.7-\mathrm{mA}$ (weak) or 1.4-mA (strong) shock of $0.5 \mathrm{sec}$ in duration. A $100-\mathrm{W}$ bulb, located behind a frosted glass plate in the ceiling of each cubicle, was operated at $60 \mathrm{~V} \mathrm{AC}$ to provide diffuse illumination of the chamber. An ambient sound level of $72 \mathrm{~dB}$ (re .0002 dynes $/ \mathrm{cm}^{2}$ ) was provided by a ventilating fan in each cubicle that operated at $55 \mathrm{~V}$ AC.

Four BRS/LVE (Model 143-26) rodent chambers, separately housed in sound-attenuating cubicles, served as the appetitiveconditioning apparatuses. The chambers measured $26.7 \times 24.8 \times$ $30.7 \mathrm{~cm}$ and had clear Plexiglas tops and sides, aluminum end walls, and a grid floor composed of $.48-\mathrm{cm}$ bars spaced $1.8 \mathrm{~cm}$ apart. A $100-\mathrm{W}$ bulb, located behind a frosted glass plate in the ceiling of each cubicle, was operated at $60 \mathrm{~V} \mathrm{AC}$ to provide diffuse illumination of the chamber. An ambient sound level of $72 \mathrm{~dB}$ was provided by a ventilating fan in each cubicle that operated at $55 \mathrm{~V} \mathrm{AC}$. A $6-\mathrm{W} 120-\mathrm{V}$ bulb, centered in the ceiling of each chamber and connected to two interval timers (Hunter Model 111-B), provided a visual CS identical to that used in aversive conditioning: a 5-sec light flashing on and off at the rate of $0.4 \mathrm{sec}$ per cycle. A BRS/LVE pellet dispenser (Model 114-20) delivered an appetitive US of four P. J. Noyes Formula A rat pellets $(45 \mathrm{mg}, 4 \mathrm{~mm}$ ) to a food magazine located in the center of the intelligence panel. To enable assessment of appetitive conditioning, the food cup was located within a small $(5 \times 7 \times 5 \mathrm{~cm})$ recessed compartment that was covered by an inward-swinging metal gate. Contact with the food cup behind the metal gate interrupted a timer that was activated with the presentation of the visual CS, thereby enabling a recording of the subject's latency in reaching the food from the onset of the CS.

\section{Procedure}

Upon receipt, all subjects were given free access to food and water for a period of 4 days. On the following day, a daily maintenance schedule of $8 \mathrm{~min}$ access to water followed by $12 \mathrm{~g}$ of food (Wayne Lab Blox) was instituted and continued for the duration of the experiment. This deprivation schedule was subsequently adjusted for water received during CER training and for food received during appetitive conditioning. Seven days following the start of their maintenance schedule, the subjects began the first of four consecutive stages of training: (1) shaping and magazine training, (2) baseline and habituation training, (3) aversive conditioning, and (4) appetitive excitatory conditioning.
Shaping and magazine training. All subjects initially received 3 days of shaping and magazine training in the appetitiveconditioning chambers, so that they would approach the food cup and eat the food pellets upon hearing the operation of the food-pellet dispenser. On the first day of training, all subjects were individually hand shaped with 25 one-pellet presentations of food. During this initial shaping session, the gate covering the recessed foodcup compartment remained open. On the second day of training, 25 one-pellet presentations of food were automatically delivered to each subject (with the gate open) on a 20-sec variable-time (VT) schedule. On the third day of magazine training, each subject received 50 one-pellet presentations of food automatically delivered on the same 20-sec VT schedule. However, during this final session, the gate to the food-cup compartment was closed so that the subject was required to push against the gate with its nose in order to reach the food cup.

Baseline and habituation training. All subjects received 6 days of baseline training in the CER chambers to establish steady licking of the drinking tube. The daily baseline sessions consisted of a 9-min drinking period, which began following the completion of an initial 10 -lick requirement. During each of the last 2 days of baseline training, the animals received habituation training consisting of two presentations of the 5-sec flashing-light CS on a 2-min VT schedule. Licks were recorded during the 5 -sec period prior to each $\mathrm{CS}$, during the $C S$, and on a minute-by-minute basis throughout the entire session. Stimulus presentations always occurred within the first $7 \mathrm{~min}$ of the 9-min session, while the subjects were exhibiting steady rates of licking. In addition, to avoid possible low preCS rates due to normal pauses in drinking, each trial was monitored and not started until the subject was licking. Trial initiation and data collection were the same in the following aversive-conditioning phase of the experiment. For a more detailed description of this CER procedure, see DeVito and Fowler (1986).

Aversive conditioning. Aversive conditioning to the 5-sec flashing-light stimulus began after habituation training. The subjects were randomly assigned to eight groups of 4 subjects each, constituting a $2 \times 4$ factorial design of two shock intensities $(0.7$ and $1.4 \mathrm{~mA}$ ) and four aversive-conditioning treatments (CS+, $\mathrm{CS}_{\mathrm{O}}$, $\mathrm{CS}-$, and $\mathrm{US}_{\mathrm{a}}$ ). Aversive conditioning lasted for 14 days, with the first 3 and the last 3 days being conducted on baseline and the intervening days being conducted off baseline. Each conditioning day involved a 9-min session during which the shock US was delivered on a 2-min VT schedule. There were two presentations of the CS and US on each of the first 2 days of aversive conditioning and four per day thereafter, for a total of 52 trials. For AvCS+ subjects, the flashing-light CS always terminated with the US, thereby generating a positive correlation of the CS and the US. For AvCSsubjects, the CS and US were explicitly unpaired (cf. Rescorla, 1967, 1969)-that is, the CS did not occur earlier than $10 \mathrm{sec}$ after or less than $30 \mathrm{sec}$ before the US, thereby generating a negative correlation of the CS and the US. For CSo subjects, a combination of the $\mathrm{CS}+$ and $\mathrm{CS}-$ schedules was employed to generate a rectangular distribution of CS-US intervals in which the CS occurred randomly from $10 \mathrm{sec}$ after the US to $5 \mathrm{sec}$ before the US. US subjects received the same temporal schedule of US presentations but without the CS. (CSo and $\mathrm{US}_{\mathrm{a}}$ controls were preferred over CS-alone and no-stimulus controls because the latter two allow a latent-inhibition effect and/or fail to control for pseudoconditioning effects. For a more complete discussion of this control-group issue, see DeVito \& Fowler, 1982.)

Appetitive excitatory conditioning. For the first 2 days of appetitive excitatory conditioning, each subject received "refresher" magazine training in which 25 one-pellet presentations of food were automatically delivered on a 2 -min VT schedule each day. During these sessions, the latency of the subject's response to the operation of the food-pellet dispenser was assessed on each trial.

Following the refresher magazine-training sessions, appetitive excitatory conditioning to the previously conditioned flashing-light 
CS commenced and continued for 20 days. On each day, each subject received five presentations of the 5-sec CS on a 2-min VT schedule, during which termination of the CS was contiguous with presentation of a four-pellet food US. During these 10-min conditioning sessions, the latency of each subject's response to the presentation of the CS was assessed on every trial.

Statistical treatment. Except where otherwise indicated, the data were uniformly subjected to a $2 \times 4$ analysis of variance (ANOVA) consisting of the two shock-US intensities $(0.7$ and $1.4 \mathrm{~mA})$ and the four aversive-conditioning treatments $(\mathrm{CS}+, \mathrm{CS}$, $\mathrm{CS}-$, and US $_{\mathrm{a}}$ ). In instances where the conditioning effect and/or the conditioning $\times$ US intensity interaction were significant at or beyond $\alpha=.05$, orthogonal polynomial contrasts were performed to (1) compare the $\mathrm{CS}_{\mathrm{O}}$ and $\mathrm{US}_{\mathrm{a}}$ controls, (2) assess a linear trend across the CS+, CS controls combined, if found to be negligibly different, i.e., $F<1$ ), and (3) evaluate residual (i.e., nonlinear trend variance). In cases where the latter was appreciable (i.e., $F>1$ ) and thus a linear trend could not be taken as the best description of the data, appropriate ancillary contrasts were performed.

\section{RESULTS}

\section{Baseline and Habituation Training}

To evaluate whether the groups were comparable prior to aversive (CER) conditioning, an assessment was made of daily licking responses during both baseline and habituation training. Separate analyses of mean daily licks over the 4 days of baseline training and over the 2 days of habituation training showed no difference between shock intensities, no differences among the aversive-conditioning treatments, and no significant interaction effect $(F \mathrm{~s}<1)$. By the last day of habituation training, the overall group mean was $1,997.3$ licks.
Assessment was also made of unconditioned suppression to the flashing-light CS during habituation training. (Suppression scores were based on the ratio, CS/[CS + preCS], where CS refers to the number of licks during the 5-sec CS and preCS refers to the number of licks during the 5-sec period prior to the CS.) Analyses of mean suppression to the CS over both days of habituation training showed no difference between shock intensities, no differences among the aversive-conditioning treatments, and no significant interaction effect $(F \mathrm{~s}<1)$. By the last trial of habituation to the CS, the overall mean suppression ratio was .41 .

\section{Aversive Conditioning}

Figure 1 presents group mean suppression ratios in twotrial blocks during the first 3 and the last 3 days of CER training. Note that means for the $\mathrm{US}_{\mathrm{a}}$ groups are not included in Figure 1 because those groups did not receive any CS presentations during CER training. Accordingly, the CER data were subjected to a $2 \times 3$ ANOVA consisting of the two shock intensities $(0.7$ and $1.4 \mathrm{~mA})$ and the three CS treatments (CS+, $\mathrm{CS}_{\mathrm{O}}$, and $\mathrm{CS}-$ ).

Although not indicated in Figure 1, there were no main or interaction effects on the first CS presentation (Trial 1), prior to the delivery of the shock US $(F \mathrm{~s}<1)$. That outcome is consistent with the lack of group differences during habituation training. However, as Figure 1 shows, differences between the shock-intensity conditions and among the CS treatments rapidly emerged and then stabilized over the course of CER training. By the end of that training, there was absolutely no suppression for the CS-

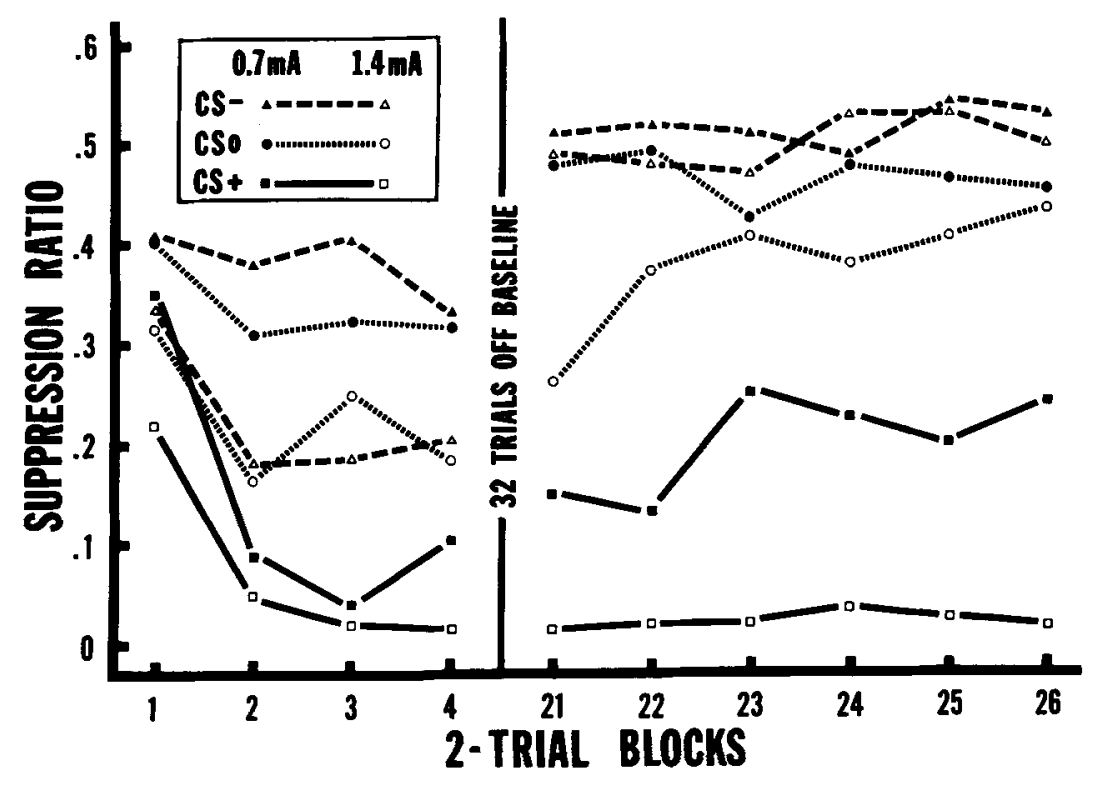

Figure 1. Mean suppression during CER training for the AvCS,$+ \mathrm{CS}_{o}$, and $\mathrm{CS}-$ groups of the $0.7-$ and 1.4-mA shock-intensity conditions. 
groups, minimal suppression for the CSo groups, and moderate to complete suppression for the CS+ groups. In addition, the increase in suppression across the CS-, $\mathrm{CS}_{\mathrm{O}}$, and $\mathrm{CS}+$ groups was considerably more pronounced for the 1.4-mA subjects than for the $0.7-\mathrm{mA}$ subjects.

Analysis of group mean suppression ratios over Trial Blocks 24-26 supports the above observations: There was a highly reliable difference between the shock intensities $[F(1,18)=21.01, p<.001]$ and among the CS treatments $[F(2,18)=110.67, p<.001]$, as well as a significant shock intensity $\times$ CS treatment interaction $[F(2,18)=6.80, p<.01]$. Trend analysis of the CS treatment effect showed both reliable linear $[F(1,18)=$ $202.43, p<.001]$ and quadratic components $[F(1,18)=$ $18.91, p<.01]$, indicating that the increase in suppression from the CS - to the CSo groups was less pronounced than the increase from the CSo to the CS+ groups. In confirmation, ancillary contrasts showed that whereas the difference between the CS- and CSo groups was reliable $[F(1,18)=11.21, p<.01]$, that between the $\mathrm{CS}_{\mathrm{O}}$ and CS+ groups was much more significant $[F(1,18)=$ $118.37, p<.001]$. In contrast, trend analysis of the CS treatment $\times$ shock intensity interaction showed an unreliable linear component $[F(1,18)=1.15, p>.05]$ but a reliable quadratic component $[F(1,18)=12.46, p<$ $.002]$. This outcome indicates that the difference between the shock-intensity treatments was due primarily to the $\mathrm{CS}+$ groups. In confirmation, ancillary contrasts showed that the difference between the $0.7-\mathrm{mA}$ and the $1.4-\mathrm{mA}$ CS + groups was highly reliable $[F(1,18)=30.56, p<$ $.001]$, whereas that between the CSo groups was unreliable $[F(1,18)=3.85, p>.05]$ and that between the CSgroups was negligible $(F<1)$.

\section{Appetitive Excitatory Conditioning}

The latency of each subject's response to the operation of the food-pellet dispenser was evaluated during the 2 days of refresher magazine training, to determine whether the groups were comparable on this measure following CER training. A $2 \times 4$ analysis (now including the US groups) of mean daily latencies over those 2 days showed no reliable effects of shock intensity, aversive-conditioning treatment, or their interaction $(F \mathrm{~s}<1)$.

Figure 2 presents, in blocks of five daily sessions, mean latencies in contacting the food cup from the onset of the 5-sec flashing-light CS for the AvCS+, CS-, and combined control (CSo and $\mathrm{US}_{\mathrm{a}}$ ) groups of the 0.7- and 1.4-mA shock-intensity conditions (left and right panels, respectively). The two control groups for each shockintensity condition were combined because, in every instance, the difference between them was negligible $(F \mathrm{~s}<1)$. As is shown in the left panel of Figure 2, the latency to contacting the food cup for the $0.7-\mathrm{mA}$ subjects decreased more rapidly for the AvCS+ group, and more slowly for the AvCS- group, relative to their CSo and $\mathrm{US}_{\mathrm{a}}$ controls. In contrast, the right panel of Figure 2 shows that the latencies for the 1.4-mA subjects decreased more rapidly for the AvCS - group, and more slowly for the AvCS+ group, relative to their controls.

A $2 \times 4$ ANOVA of mean latencies over the first half of appetitive conditioning (Blocks 1 and 2) showed negligible main effects of shock intensity and CS treatment $\left(F_{\mathrm{s}}<1\right)$, but a reliable effect of their interaction $[F(3,24)=3.95, p<.025]$. Trend analysis of the interaction indicated a highly significant linear component $[F(1,24)=11.25, p<.005]$, which accounted for virtually all $(95.1 \%)$ of the interaction variance. Thus, there

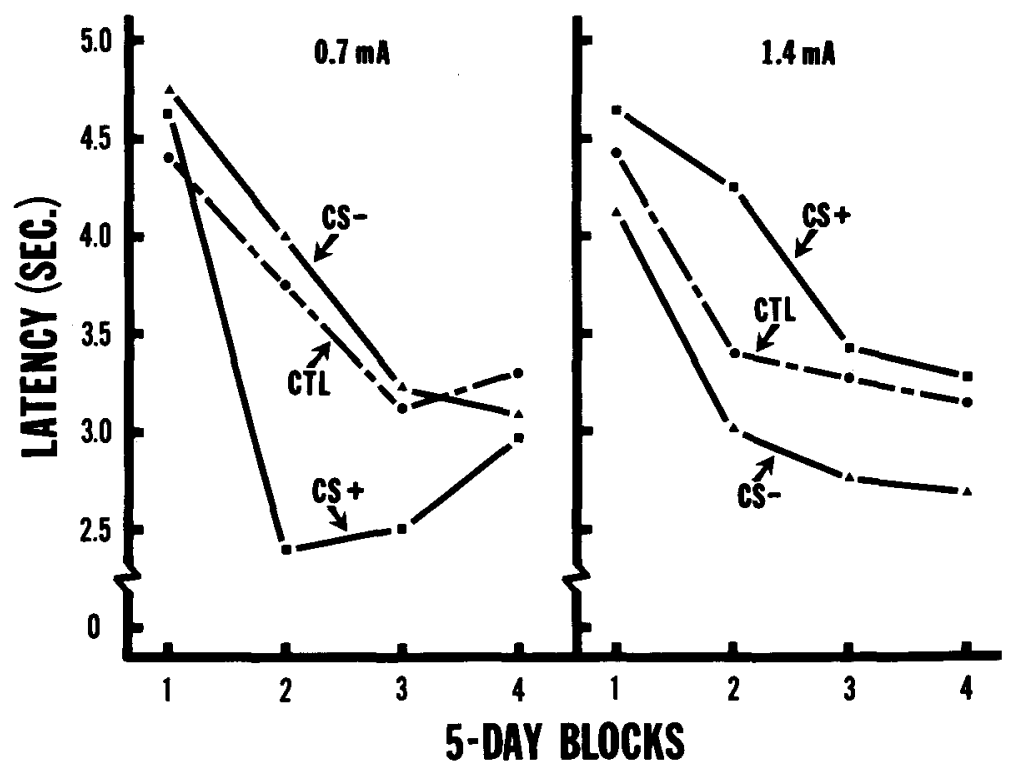

Figure 2. Mean latency in contacting the food cup from the onset of the CS for the AvCS,$+ \mathrm{CS}-$, and combined control $\left(C T L=C_{5}\right.$ and $U_{\text {a }}$ ) groups of the 0.7 and 1.4-mA shock-intensity conditions (left and right panels, respectively). 
was negligible residual (i.e., nonlinear) trend variance and no interaction effect for the CSo and $\mathrm{US}_{\mathrm{a}}$ control groups of the two shock-intensity conditions $(F s<1)$. Given these results, the best description of the data is that the latency scores across the CS+, control, and CS- treatments increased linearly for the $0.7-\mathrm{mA}$ subjects and decreased linearly for the 1.4-mA subjects. In confirmation, separate assessments by polynomial contrasts of the latency scores for each shock-intensity condition showed that, in both cases, there was a reliable linear effect across the CS+, control, and CS- groups $(F \mathrm{~s} \geq 5.32$, ps < $.05)$, with nonlinear trend variance and the difference between the CS $_{o}$ and US $_{a}$ control groups of each set being negligible $\left(F_{\mathrm{s}}<1\right)$. In addition, the difference between the two sets of controls for the 0.7- and the 1.4-mA shockintensity conditions was negligible, as was the difference between the 0.7-mA CS+ and 1.4-mA CS- groups, which showed facilitated performance relative to the controls, and the difference between the $0.7-\mathrm{mA} \mathrm{CS}-$ and the 1.4$\mathrm{mA} \mathrm{CS}+$ groups, which showed retarded performance relative to the controls (all $F \mathrm{~s}<1$ ). Collectively, these results indicate that the facilitated or retarded transfer effects for the CS+ and CS- groups were of comparable magnitude both within and across the 0.7- and 1.4-mA shock-intensity conditions. ${ }^{1}$

In contrast to the ANOVA results for the first half of appetitive conditioning, those for the second half (Blocks 3 and 4) showed no reliable main or interaction effects. These results for the second half are to be expected in that all of the subjects learned to rapidly approach the food cup by the end of appetitive conditioning: In effect, the groups that were retarded during the first half of appetitive conditioning "caught up" to those groups that were facilitated during the first half.

\section{DISCUSSION}

The results of this study indicate that positive and negative transfer effects in an Av-to-Ap reconditioning paradigm are dependent on the intensity of the US that is employed in aversive conditioning. For those groups that were pretrained with the weak 0.7-mA shock US, appetitive reconditioning of the CS was facilitated for the AvCS + subjects and retarded for the AvCS- subjects relative to their $\mathrm{CS}_{\mathrm{O}}$ and $\mathrm{US}_{\mathrm{a}}$ controls. In contrast, for those groups that were pretrained with the strong 1.4-mA shock US, appetitive reconditioning of the CS was retarded for the AvCS+ subjects and facilitated for the AvCS- subjects relative to their $\mathrm{CS}_{\mathrm{o}}$ and $\mathrm{US}_{\mathrm{a}}$ controls.

The present findings are consistent with our affectivebehavioral-cognitive interpretation of across-reinforcement transfer effects. This interpretation posits that, although the rate of reconditioning in a transfer paradigm is positively related to the size of the affective discrepancy between the outcome that the preestablished CS predicts and that which the current US actually provides, this effect can be offset and even reversed by the magnitude of the behavioral discrepancy that prevails between the type of response that is initially conditioned to the CS and that which is required in the transfer test. Therefore, it follows that, in the present transfer paradigm, Ap conditioning for the AvCS + subjects would generate a large affective discrepancy (predicted bad but obtained good outcome) and thus facilitate conditioning, whereas Ap conditioning for the AvCS - subjects would generate a small affective discrepancy (predicted good and obtained good outcome) and thus retard conditioning. Accordingly, these affective discrepancies should produce positive transfer effects in the case of the $0.7-\mathrm{mA}$ subjects, for which the responses initially conditioned to the $\mathrm{AvCS}+, \mathrm{CS}_{\mathrm{o}}$, and CS- were grossly comparable (i.e., minimal to no suppression) and therefore provided little behavioral disparity with the ambulatory response required in Ap reconditioning (i.e., approach to the food cup). However, in the case of the 1.4-mA subjects, where the responses initially conditioned to the AvCS+, CSo, and CS- were grossly different (i.e., from pronounced to no suppression), there would be marked behavioral discrepancies for the groups relative to the response required in Ap reconditioning and, as a result, they could override the affective discrepancy and generate negative transfer effects.

In the present study, an assessment of suppression ratios was used to provide evidence concerning the behavioral disparities between Av and Ap conditioning that are produced by weak and strong shock USs. Although sufficient to demonstrate differences between the shockintensity conditions, this measure does not elucidate the nature of the responses that were established in Av conditioning. We did not make systematic observations of the specific kinds of behaviors that were elicited by the AvCSs, but our casual observations of the animals indicated that, upon receipt of the flashing-light CS, the 1.4-mA CS + subjects turned away from the drinking tube toward the center of the chamber and cringed in anticipation of the shock; in contrast, the 1.4-mA CS- subjects remained undisturbed at the drinking tube and continued to lick, whereas the 1.4-mA CSo subjects acted in an intermediate fashion. This range of behaviors was considerably constrained for the $0.7-\mathrm{mA}$ animals in that the CS+ subjects of this condition acted more like the 1.4-mA CSo subjects. These observations are consistent with the findings of other CER studies that have employed direct observations of the rat's behavior. For example, Bevins and Ayres (1992) found that, for rats trained with a 2-min light CS and a 0.8-mA shock US that was twice the duration of the shock used in the present study (and, hence, more comparable to our 0.5-sec 1.4-mA shock), a CS + caused the rat to freeze occasionally or to move from the dipper mechanism and lever manipulandum to the center and rear of the chamber; in contrast, a CScaused no systematic changes in the rat's location and no disruption of its operant behavior.

The findings of other studies that have investigated the effects of weak and strong shock USs in producing different kinds of conditioned responses (CRs) to a CS are also in line with our interpretation. For example, Hol- 
land (1979, Experiment 3) has shown through the use of direct behavioral observations that weak shock USs support CRs (e.g., rearing to a light CS) that have components in common with CRs supported by a food US (i.e., rearing to a light $C S$ ); in contrast, strong shock USs support CRs (e.g., freezing to a light CS) that have no obvious components in common with a food-based CR.

The demonstration of positive Av-to-Ap transfer for the $0.7-\mathrm{mA}$ subjects of the present study is important because it is, to our knowledge, the first and only demonstration of its kind in the over 30-year history of acrossreinforcement transfer effects. Indeed, as previously reviewed, the extant findings on Av-to-Ap transfer have uniformly shown negative transfer. We believe, however, that the present demonstration of both positive and negative transfer, based on the use of weak and strong shock USs, respectively, affords a straightforward interpretation of the negative transfer typically found in this paradigm: In all of the previous studies of Av-to-Ap transfer, relatively strong shock USs have been employed in the aversive-conditioning phase of the study. Consequently, as observed for the 1.4-mA subjects of the present study, those strong shocks would generate large behavioral discrepancies between the CRs established in Av and Ap conditioning and, hence, the basis for a negative transfer effect.

As previously noted, our interpretation readily handles the positive transfer that has been reported in Ap-to-Av transfer studies (e.g., Bromage \& Scavio, 1978; DeVito \& Fowler, 1982). This is so because transfer in this paradigm should be primarily influenced by the affective discrepancy between what the ApCS predicts and what the AvUS affords, inasmuch as behavioral differences among the Ap conditioning groups are relatively small (e.g., general locomotion as opposed to approaching the food cup) and thus provide similar disparities with the target behavior in Av conditioning. As for the instances of negative transfer found in this paradigm (Dickinson, 1976; Konorski \& Szwejkowska, 1956), we have previously argued (DeVito \& Fowler, 1982) that these studies, despite their seemingly clear outcomes, are subject to certain methodological and/or procedural problems. For example, the negative transfer reported in Konorski and Szwejkowska's classic study was based on the performance of a single dog who had a long history of both conditioning and extinction with the ApCS+ and ApCS- stimuli used in the Av reconditioning test. In Dickinson's study, which involved two experiments, an ApCS+ group actually showed positive transfer in both experiments relative to an ApCSo control, but negative transfer relative to a USalone control that was employed in only the first experiment. That US-alone control, however, did not have any prior exposure to the CS (e.g., habituation training), and therefore it may have benefited from a salient novel CS in Av conditioning.

The findings of null transfer that have been reported for Ap-to-Av paradigms (Jackson, 1974; Peck \& Bouton, 1990 , Experiment 3) would also seem attributable to cer- tain methodological features. For example, in Jackson's study, the lack of reliable differences among the ApCS + , CSo, CS-, and CS-alone groups may have been due to the fact that a CER procedure entailing a food-reinforced baseline behavior was used, which may have interacted with the food-reinforced ApCSs used to signal shock in CER training, thereby obscuring a positive transfer effect (see DeVito \& Fowler, 1982). In Peck and Bouton's (1990) study, which used a direct behavioral observation technique to assess the responses elicited by the CS, it was found that prior ApCS- training retarded reconditioning of the CS as a signal for shock, but prior ApCS+ training did not facilitate such reconditioning relative to a novel-CS control group. Although Peck and Bouton actually show both positive and null transfer (for their ApCS - and ApCS+ groups, respectively), the latter outcome may, like Dickinson's (1976) earlier finding, be the result of the use of a novel and, hence, more salient CS for the untrained control group. Whatever the reasons for these disparate findings in Ap-to-Av transfer, it is clear that the present demonstration of both positive and negative Av-to-Ap transfer, based on the use of weak and strong shock USs to differentially condition suppression to the CS, argues strongly for the role of behavioral, as well as affective and cognitive, factors in the control of across-reinforcement transfer effects.

\section{REFERENCES}

Bevins, R. A., \& AYres, J. J. B. (1992). Rat's location during conditioned suppression training. Animal Learning \& Behavior, 20, 8-16. BromaGe, B. K., \& SCAVIo, M. J., JR. (1978). Effects of an aversive CS+ and CS- under deprivation upon successive classical appetitive and aversive conditioning. Animal Leaming \& Behavior, 6, 57-65.

DEVITo, P. L., \& FowLER, H. (1982). Transfer of conditioned appetitive stimuli to conditioned aversive excitatory and inhibitory stimuli. Learning \& Motivation, 13, 135-154.

DEVITO, P. L., \& Fow LER, H. (1986). Effects of contingency violations on the extinction of a conditioned fear inhibitor and a conditioned fear excitor. Journal of Experimental Psychology: Animal Behavior Processes, 12, 99-115.

Dickinson, A. (1976). Appetitive-aversive interactions: Facilitation of aversive conditioning by prior appetitive training in the rat. Animal Learning \& Behavior, 4, 416-420.

Dickinson, A., \& Pearce, J. M. (1977). Inhibitory interactions between appetitive and aversive stimuli. Psychological Bulletin, 84, 690-711.

FoWLER, H. (1978). Cognitive associations as evident in the blocking effects of response contingent CSs. In S. H. Hulse, H. Fowler, \& W. K. Honig (Eds.), Cognitive processes in animal behavior (pp. 109153). Hillsdale, NJ: Erlbaum.

Fowler, H., LySLE, D. T., \& DEVito, P. L. (1991). Conditioned excitation and conditioned inhibition: Asymmetric processes as evident in extinction. In M. R. Denny (Ed.), Fear, avoidance, and phobias (pp. 317-362). Hillsdale, NJ: Erlbaum.

Holland, P. C. (1979). The effects of qualitative and quantitative variation in the US on individual components of Pavlovian appetitive conditioned behavior in rats. Animal Learning \& Behavior, 7, 424-432.

JACKsoN, D. E. (1974). CS-free food contingencies and subsequent acquisition of conditioned suppression: No transfer effect. Bulletin of the Psychonomic Society, 4, 235-236.

Kaye, H., Preston, G. C., Szabo, L., Druiff, H., * Mackintosh, N. J. (1987). Context specificity of conditioning and latent inhibition: Evidence for a dissociation of latent inhibition and associative 
interference. Quarterly Journal of Experimental Psychology, 39B, 127-145.

KoNORSKI, J., \& SzWEJKowSKA, G. (1956). Reciprocal transformations of heterogeneous conditioned reflexes. Acta Biologiae Experimentalis, 17, 141-165.

Krank, M. D. (1985). Asymmetrical effects of Pavlovian excitatory and inhibitory aversive transfer on Pavlovian appetitive responding and acquisition. Learning \& Motivation, 16, 35-62.

PECK, C. A., \& Bouton, M. E. (1990). Context and performance in aversive-to-appetitive and appetitive-to-aversive transfer. Learning \& Motivation, 21, 1-31.

Rescorla, R. A. (1967). Pavlovian conditioning and its proper control procedures. Psychological Review, 74, 71-80.

Rescorla, R. A. (1969). Pavlovian conditioned inhibition. Psychological Bulletin, 72, 77-94.

Rescorla, R. A., \& Wagner, A. R. (1972). A theory of Pavlovian conditioning: Variations in the effectiveness of reinforcement and nonreinforcement. In A. H. Black \& W. F. Prokasy (Eds.), Classical conditioning II: Current research and theory (pp. 64-99). New York: Appleton-Century-Crofts.

SCAvio, M. J., JR. (1974). Classical-classical transfer: Effects of prior aversive conditioning upon appetitive conditioning in rabbits (Oryctolagus cuniculus). Joumal of Comparative \& Physiological Psychology, 86, 107-115.

SCAvio, M. J., JR., \& Gormezano, I. (1980). Classical-classical transfer: Effects of prior appetitive conditioning upon aversive conditioning in rabbits. Animal Learning \& Behavior, 8, 218-224.

\section{NOTE}

1. The above trend analysis focuses on the interaction of US intensity with the entire dimension of CS/US correlation, and not just with a positive or a negative CS/US contingency. Nevertheless, one can ask whether there was a reliable difference between the controls and either the facilitated transfer groups $(0.7-\mathrm{mA} \mathrm{CS}+$ and $1.4-\mathrm{mA} \mathrm{CS}-)$ or the retarded transfer groups (0.7-mA CS- and 1.4-mA CS+), even though such an assessment effectively partitions the data in half and, therefore, calls for a conclusion based on restricted data sets. Despite those restrictions, the results of such a partitioning were favorable in that they indicated, at least, a marginally reliable difference between the controls and both the facilitated groups $[F(1,24)=3.55, p<.08]$ and the retarded groups $[F(1,24)=3.96, p<.06]$. In this instance, one must guard against the possibility of a Type II error-that is, concluding that there was no difference between the controls and either the facilitated or the retarded groups, because together those judgments can foster the overall conclusion of no differences across the entire dimension of CS/US correlation, in clear contradiction to the highly significant results of the trend analysis. Indeed, when the same partitioning was applied to the second block of appetitive conditioning trials, so as to eliminate early trial variance, the results showed that the difference between the controls and, at least, the facilitated groups was unquestionably reliable $[F(1,24)=8.84, p<.01]$.

(Manuscript received May 17, 1993;

revision accepted for publication November 3, 1993.) 